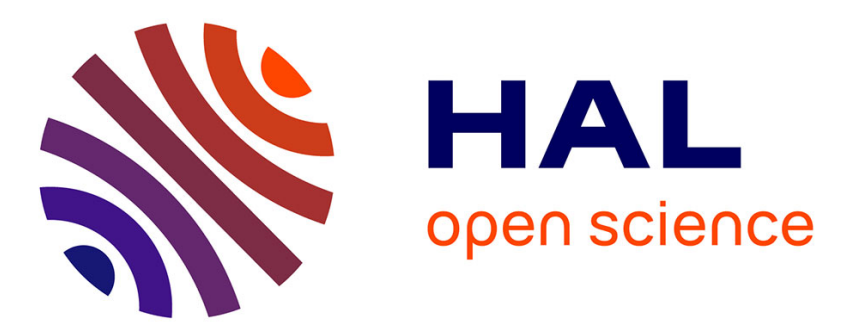

\title{
Institutionalisierung der Freiheit im Recht. Foucault und die postmarxistische Rechtskritik
}

\author{
Karsten Schubert
}

\section{To cite this version:}

Karsten Schubert. Institutionalisierung der Freiheit im Recht. Foucault und die postmarxistische Rechtskritik. Steffen K. Herrmann; Matthias Flatscher. Institutionen des Politischen, pp.351 - 378, 2020, 10.5771/9783748904618-351 . hal-02989814

\section{HAL Id: hal-02989814 https://hal.science/hal-02989814}

Submitted on 5 Nov 2020

HAL is a multi-disciplinary open access archive for the deposit and dissemination of scientific research documents, whether they are published or not. The documents may come from teaching and research institutions in France or abroad, or from public or private research centers.
L'archive ouverte pluridisciplinaire HAL, est destinée au dépôt et à la diffusion de documents scientifiques de niveau recherche, publiés ou non, émanant des établissements d'enseignement et de recherche français ou étrangers, des laboratoires publics ou privés. 
Schubert, Karsten (2020): Institutionalisierung der Freiheit im Recht. Foucault und die postmarxistische Rechtskritik. In: Steffen K. Herrmann und Matthias Flatscher (Hg.): Institutionen des Politischen. Baden-Baden: Nomos, S. 351-378. https://doi.org/10.5771/9783748904618-351

Unkorrigiertes Manuskript! Wenn Sie keinen Zugang zur publizierten Version haben, wenden Sie sich gerne per Email an mich.

\title{
Institutionalisierung der Freiheit im Recht. Foucault und die postmarxistische Rechtskritik
}

\author{
Karsten Schubert
}

\begin{abstract}
In der postmarxistischen politischen Philosophie wird die Veränderung gegenüber der Verstetigung analytisch und normativ bevorzugt. Deshalb leistet die postmarxistische Theorie keine realistische Analyse und normativ-differenzierende Bewertung von politischen Institutionen, insbesondere des Rechts. Der Beitrag liefert einen Ansatz, um diese Lücke zu schließen, indem er der Abblendung von institutionellen Fragen mit einer neuen postfundamentalistischen Institutionentheorie begegnet. Diese Theorie basiert auf dem Freiheitsbegriff der Kritik, der im Anschluss an Foucault entwickelt wird und zeigt, dass kritischer Subjektivität durch die Institutionalisierung von freiheitlichen Subjektivierungsregimen bedingt ist. Dieser Freiheitsbegriff korrigiert den avanciertesten Ansatz der postmarxistischen Rechtstheorie, Menkes Rechtskritik. Deren Stärke ist es, die liberal entpolitisierende Subjektivierung des Rechts zu kritisieren, doch Menkes Vorschlag einer radikalen Politisierung ist paternalistisch. Mit Foucault lässt sich hingegen ein Recht denken, das Subjektivierung kritisiert und dennoch nicht paternalistisch ist, weil es die Subjekte zur Kritik der Institutionen befähigt.
\end{abstract}

\section{Einleitung}

Die postmarxistische politische Philosophie ist eine Philosophie der Veränderung und des Werdens. Postmarxistisch ist sie, weil sie keine allgemeinen Gesetzmäßigkeiten und Ziele des Werdens kennt ökonomischer Determinismus und Teleologie sind passé - und Versuche 
der Rekonstruktion universeller Vernunft verdächtigt sie, Vehikel partikularer (Klassen-)Interessen zu sein.

In der Debatte um ,das Politische', die ,politische Differenz' und den ,Postfundamentalismus‘ wurde die postmarxistische Philosophie der Veränderung in den letzten Jahren mit systematischer Schärfe formuliert und sozialontologisch fundiert. ${ }^{1}$ Die Debatte stellt Kontingenz und Konfliktualität als zentrale Eigenschaften des Politischen heraus. Sie zeigt dadurch, dass verstetigte und institutionalisierte Ordnung immer das Resultat von Kämpfen um Hegemonie ist, bei denen es Gewinner und Verlierer gibt. Verstetigte Ordnung und Institutionen erzeugen deshalb Ausschlüsse. Die Frage, wie eine ,gute Ordnung' und ,gute Institutionen zu denken sind, wurde in Anbetracht dieser Diagnose zugunsten der

1 Grundlegende Primärliteratur in dieser Debatte sind u. a. Laclau, Ernesto/Mouffe, Chantal: Hegemonie und radikale Demokratie. Zur Dekonstruktion des Marxismus. 2. Auflage. Übers. von Michael Hintz und Gerd Vorwallner. Wien: Passagen 2001; Lefort, Claude/Gauchet, Marcel: „Über die Demokratie: Das Politische und die Instituierung des Gesellschaftlichen“, in: Rödel, Ulrich (Hg.): Autonome Gesellschaft und libertäre Demokratie, Frankfurt a. M.: Suhrkamp 1990, 89-122; Lefort, Claude: „Die Frage der Demokratie“, in: Rödel, Ulrich (Hg.): Autonome Gesellschaft und libertäre Demokratie. Frankfurt am Main: Suhrkamp 1990, 281-297; Mouffe, Chantal: Über das Politische. Wider die kosmopolitische Illusion. Übers. von Niels Neumaier. Frankfurt am Main: Suhrkamp 2007; Rancière, Jaques: Das Unvernehmen. Politik und Philosophie. Übers. von Richard Steurer. Frankfurt am Main: Suhrkamp 2002 und Hardt, Michael/Negri, Antonio: Empire. Die neue Weltordnung. Übers. von Thomas Atzert und Andreas Wirthensohn. Frankfurt am Main: Campus 2002. Siehe für die Umfangreiche deutsche Debatte dazu in den Sammelbänden Flügel, Oliver/Heil, Reinhard/Hetzel, Andreas (Hg.): Die Rückkehr des Politischen. Demokratietheorien heute. Darmstadt: Wissenschaftliche Buchgesellschaft 2004; Heil, Reinhard/Hetzel, Andreas (Hg.): Die unendliche Aufgabe. Kritik und Perspektiven der Demokratietheorie. Bielefeld: transcript 2004; Bedorf, Thomas/Röttgers, Kurt (Hg.): Das Politische und die Politik. Berlin: Suhrkamp 2010 und Bröckling, Ulrich/Feustel, Robert (Hg.): Das Politische denken. Zeitgenössische Positionen. Bielefeld: transcript 2010. Einen klaren philosophisch-systematischen Zugang zum in diesen Beiträgen verhandelten Problemfeld entwickelt Oliver Marchart unter dem Schlagwort ,Postfundamentalismus‘, vgl. Marchart, Oliver: „Politische Theorie als Erste Philosophie. Warum der ontologischen Differenz die politische Differenz zugrunde liegt“, in: Bedorf, Thomas/Röttgers, Kurt (Hg.): Das Politische und die Politik. Berlin: Suhrkamp 2010, 143-158 und ausführlicher ders.: Die politische Differenz. Zum Denken des Politischen bei Nancy, Lefort, Badiou, Laclau und Agamben. Berlin: Suhrkamp 2011 und ders.: Das unmögliche Objekt. Eine postfundamentalistische Theorie der Gesellschaft. Berlin: Suhrkamp 2013. 
Theoretisierung der Veränderung und des Umbruchs vernachlässigt. Insbesondere gibt es in diesem postfundamentalistischen Debattenfeld keine Theorie des Rechts. ${ }^{2}$ Eine solche Theorie müsste das Verhältnis zwischen Recht und Politik, zwischen rechtlicher Verfestigung und politischer Veränderung problematisieren und dabei fragen, wie politische Veränderung im Recht möglich ist und wieviel rechtliche Festschreibung von Politik nötig ist.

Im Feld der postmarxistischen Rechtstheorie gab es zuletzt große Bewegung durch die Publikation von Christoph Menkes Kritik der Rechte. Menke hat zum Ziel, einen neuen Rechtsbegriff zu entwickeln, mit dem sich Recht als Sphäre der Förderung von politischer Veränderung denken lässt. Insofern Menke prima facie die Frage nach Ordnung und Institution im gemeinsamen Paradigma des Werdens und der Veränderung stellt, ist die Erwartung berechtigt, dass seine Theorie mobilisiert werden könne, um das rechtstheoretische und institutionelle Defizit des Postfundamentalismus zu beheben. Wie ich im Folgenden zeigen werde, wird diese Erwartung aber enttäuscht, weil Menke die politische Veränderung gegen rechtliche Fixierungen und Entpolitisierungen positioniert. So führt auch Menkes Rechtskritik nicht zu einer Theorie der Institutionalisierung von Veränderung im Recht und bleibt bezüglich der Frage nach guter rechtlicher Ordnung und ihren Institutionen genauso stumm wie die postfundamentalistische Werdensphilosophie.

Dennoch enthalten sowohl die Debatte um den Postfundamentalismus als auch Menkes Rechtskritik schon den zentralen Gedanken zur Beantwortung der Frage nach guter Ordnung und guten Institutionen im Postmarxismus: Gute Ordnung ist die Verstetigung der Veränderung, die Institutionalisierung der Instituierung. Meine These ist, dass dieses komplexe Verhältnis mit einer Reaktualisierung des Freiheitsbegriffs

2 Im Anschluss an Luhmann und teilweise Derrida gibt es eine sehr produktive rechtstheoretische Debatte, die postfundamentalistisch in einem weiten Verständnis ist, allerdings keine Verbindung zum hier gemeinten Postfundamentalismus hat, der um die Erläuterung des Begriffs des Politischen bemüht ist. Vgl. dazu Gunther Teubner: „Ökonomie der Gabe - Positivität der Gerechtigkeit: Gegenseitige Heimsuchungenvon System und différance“, in: Albrecht Koschorke/Cornelia Vismann (Hg.): Widerstände der Systemtheorie. Kulturtheoretische Analysen zum Werk von Niklas Luhmann 1999, Berlin: Akademie Verlag 1999, 199-212; Gunther Teubner (Hg.): Nach Jacques Derrida und Niklas Luhmann: zur (Un-)Möglichkeit einer Gesellschaftstheorie der Gerechtigkeit. Stuttgart: Lucius \& Lucius 2008 und Andreas Fischer-Lescano: Globalverfassung. Die Geltungsbegründung der Menschenrechte. Weilerswist: Velbrück Wissenschaft 2005. 
beschrieben werden kann. Freiheit, verstanden als kontinuierliche selbstreflexive Kritik, ist derjenige normative Begriff, der sich aus der postfundamentalistischen Sozialontologie von Konflikt und Kontingenz herleiten lässt. Er ist auch die systematische Antwort auf die Pathologien der Entpolitisierung durch das bürgerlich-liberale Recht, die Menke analysiert. Freiheit als Kritik kann dabei einerseits das Operieren von Recht und Politik liberal-pluralistischen Demokratien beschreiben, und andererseits als normativer Kritikbegriff für die Analyse ihrer Dysfunktionalität dienen.

Um diese These zu begründen, gehe ich zurück zu einem Theoretiker, der eine Grundlage der aktuellen Debatte um das Politische und den Postfundamentalismus bildet: Foucault, dessen Machttheorie als Ansatz einer Sozialontologie der Kontingenz und Konfliktualität gelten kann. Gleichzeitig hat Foucault den Begriff der Freiheit als den zu dieser Ontologie passenden normativen Begriff ausgezeichnet. Meine Intervention besteht erstens darin, den Begriff der Freiheit als Kritik systematisch herauszuarbeiten und dabei - mit Foucault gegen Foucault $\mathrm{zu}$ zeigen, dass er nur innerhalb einer pluralistisch-demokratischen Institutionentheorie gedacht werden kann.

Das Recht spielt in einer solchen Institutionentheorie eine zentrale Rolle. Nach der systematischen Herleitung des Freiheitsbegriffs der Kritik und seiner Funktion als Institutionalisierung der Instituierung werden ich mich deshalb zweitens der Institution des Rechts zu. Dabei zeige ich, wie der Foucault'sche Freiheitsbegriff Menkes Rechtskritik korrigiert und so zur Analyse und Kritik des Verhältnisses von Recht und Politik dienen kann. Kritisch anschließend an Menkes Versuch, ein „neues Recht“ der Repolitisierung zu denken, argumentiere ich, dass liberale subjektive Rechte eine ambivalente Rolle spielen: Die liberale Subjektivierung durch subjektive Rechte führt einerseits zur Essentialisierung der liberalbürgerlichen Ordnung. Dennoch sind sie unverzichtbar, um zu verhindern, dass die politische Kritik der Subjektivierung zu Paternalismus führt. Die nach Foucault konzipierte Institutionalisierung der Institutierung bleibt so ein Geschäft der kritischen Subjekte und subjektive Rechte sind Teil des Subjektivierungsregimes, das Kritik ermöglicht.

\section{Freiheit und Institutionen nach Foucault}

Ich schildere nun - sehr schematisch - den Aufbau des Arguments für Freiheit als Kritik. Die folgenden Argumentationsschritte sind das Ergebnis 
einer Rekonstruktion und Kritik der sozialphilosophischen Debatte um Foucaults Freiheitsbegriff, die das Fleisch meiner Monographie Freiheit als Kritik. Sozialphilosophie nach Foucault bildet. ${ }^{3}$ Für die systematische Prägnanz beschränke ich mich hier auf das argumentative Skelet.

Subjektivierung bedeutet, dass Subjekte durch Macht konstituiert sind. Macht ist produktiv, weil sie es den Subjekten ermöglicht, zu denken und zu handeln; das heißt aber, dass es keine Eigenschaften des Subjekts gibt, von denen man sicher sagen kann, dass sie nicht durch Macht im Prozess der Subjektivierung instantiiert worden sind. Insbesondere können keine anspruchsvollen Fähigkeiten als der Subjektivierung vorgängig im Subjekt angenommen werden. Zwar subjektivieren sich Subjekte auch selbst, Subjektivierung ist also kein passives Erleiden des Subjekts, sondern ein aktiver Vorgang des sich selbst Subjektivierens. Doch die dazu verwendeten Selbsttechnologien stehen auch in dem Verdacht, nicht vom Subjekt selbst gebildet, sondern von Macht instantiiert zu sein. Subjektivierung ist ein Freiheitsproblem, weil sie zu der begründeten Befürchtung führt, dass Subjekte viel fremdbestimmter sind, als gemeinhin angenommen. Diese Situation stellt den Freiheitsbegriff vor ein Problem: Freiheit kann nicht mehr negativ in der Nichteinmischung bestehen, weil es keinen eigenen und inneren Kern des Subjekts gibt, der vom Verdacht der Vermachtung ausgenommen wäre. Freiheit kann auch nicht positiv in der einfachen Übernahme und Anerkennung der subjektivierenden Macht Interpretationsstrategien von Foucaults Werk in Bezug auf das Freiheitsproblem anhand von exemplarischen Vertreter_innen dieser Strategien und leite durch eine interne Kritik dieser Texte den Begriff der Freiheit als Kritik her. Die vier Strategien und ihre Vertreter_innen sind: 1. Foucault ist kohärent (Patton, Paul: „Taylor and Foucault on Power and Freedom“, in: Political Studies 37 (2), 1989, 260-276.), 2. Foucault korrigiert sich (Lemke, Thomas: Eine Kritik der politischen Vernunft. Foucaults Analyse der modernen Gouvernementalität. Hamburg: Argument 1997), 3. Foucault kritisiert kohärent (Saar, Martin: Genealogie als Kritik. Geschichte und Theorie des Subjekts nach Nietzsche und Foucault. Frankfurt: Campus 2007), 4. Foucault ist nicht genug (Allen, Amy: The Politics of Our Selves. Power, Autonomy, and Gender in Contemporary Critical Theory. New York: Columbia University Press 2008). Der Text dieses Abschnitts basiert auf dem Kapitel „Das Argument“ in Schubert: Freiheit als Kritik, S. 305-312. 
bestehen, weil diese oft unterwerfende Effekte hat. ${ }^{4}$ Zwar ist Macht nach Foucault produktiv, doch dies lässt offen, ob ihre produktiven Effekte repressiv oder freiheitlich sind. ${ }^{5}$ Das Freiheitsproblem der Subjektivierung besteht in der Frage, wie Freiheit in dieser Situation der Äquidistanz zu einem negativen und positiven Freiheitsbegriff gedacht werden kann.

Freiheit in Bezug auf das Freiheitproblem der Subjektivierung bedeutet die Fähigkeit zur reflexiven Kritik der eigenen Subjektivierung. ${ }^{6}$ Diese genealogischer Phase formuliert mit den Hauptwerken Foucault, Michel: Wahnsinn und Gesellschaft. Eine Geschichte des Wahns im Zeitalter der Vernunft. Übers. von Ulrich Köppen. Frankfurt am Main: Suhrkamp 1973; ders.: Die Ordnung der Dinge. Eine Archäologie der Humanwissenschaften. Übers. von Ulrich Köppen. Frankfurt am Main: Suhrkamp 1974; ders.: Die Ordnung des Diskurses. 9. Auflage. Übers. von Walter Seitter. Frankfurt am Main: Fischer 1991; ders.: Überwachen und Strafen. Die Geburt des Gefängnisses. Übers. von Walter Seitter. Frankfurt am Main: Suhrkamp 1994. Umfangreiche Verweise auf Sekundärliteratur zur Interpretation Foucaults und zur Konstruktion von Freiheitsproblem und Freiheitsbegriff finden sich in Schubert: Freiheit als Kritik.

5 Den produktiven Machtbegriff entwickelt Foucault insbesondere in Foucault: Überwachen und Strafen und ders.: Der Wille zum Wissen. Sexualität und Wahrheit 1. Übers. von Ulrich Raulff und und Walter Seitter. Frankfurt am Main: Suhrkamp 1983; die Ambivalenz der produktiven Macht wurde insbesondere bezüglich Foucaults Vorlesungen zur Gouvernementalität und zum Regieren diskutiert: Foucault, Michel: Sicherheit, Territorium, Bevölkerung. Geschichte der Gouvernementalität I. Übers. von Claudia BredeKondersmann. Frankfurt am Main: Suhrkamp 2004; ders.: Die Geburt der Biopolitik. Geschichte der Gouvernementalität II. Übers. von Jürgen Schröder. Frankfurt am Main: Suhrkamp 2006; ders.: Die Regierung des Selbst und der anderen. Vorlesung am Collège de France 1982/83. Übers. von Jürgen Schröder. Berlin: Suhrkamp 2009.

6 Dieser Freiheitsbegriff findet sich vor allem in Foucaults späten Reflexionen auf seine Methode der genealogischen Kritik, u.a. in Foucault, Michel: Was ist Kritik? Übers. von Walter Seitter. Berlin: Merve 1992; ders.: „Was ist Aufklärung?“, in: ders.: Schriften in vier Bänden. Dits et Ecrits - Band IV. 19801988. Hg. von Daniel Defert und François Ewald und übers. von Michael Bischoff. Frankfurt am Main: Suhrkamp 2005, 687-707; ders.: „Was ist Aufklärung? Auszug aus der Vorlesung vom 5. Januar 1983“, in: ders.: Schriften in vier Bänden. Dits et Ecrits - Band IV. 1980-1988. Hg. von Daniel Defert und François Ewald und übers. von Michael Bischoff. Frankfurt am Main: Suhrkamp 2005, 837-848. Siehe dazu auch Butler, Judith: „Was ist Kritik. Ein Essay über Foucaults Tugend“, in: Deutsche Zeitschrift für Philosophie 50 (2), 2002, 249265. Die übliche Foucault Rezeption kritisierend weise ich darauf hin, dass der 
Fähigkeit ermöglicht es, mit Subjektivierung als Freiheitsproblem umzugehen. Sie ist eine höherstufige Reflexion im Gegensatz zu der einfach durch Subjektivierung gestifteten Reflexion, die es ermöglichen kann, die von der Subjektivierung instantiierte Reflexion zu überschreiten. Anders gesagt ist sie eine bestimmte Selbsttechnologie, deren Operation die Reflexion der potentiellen Vermachtung aller Selbsttechnologien ist. Zwar ist auch Freiheit als Kritik von der Subjektivierung abhängig - ,absolute‘ Freiheit oder Freiheit als einen festgesetzten Status wird es also nicht geben können -, doch als immer über sich selbst hinauszielende Bewegung erreicht sie so viel Distanz zur und Eigenständigkeit gegenüber der Subjektivierung wie möglich. Sie ist eine internalisierte, ständige Hermeneutik des Verdachts, die immer alles, und auch sich selbst, kritisch überprüft. Dabei kommt sie zu keinem Stillstand, sondern kann nur kritische Operation an kritische Operation anschließen; sie ist deshalb eine Praxis (und kein Status oder Zustand), die aber dennoch von Fähigkeiten abhängig ist.

Durch diese Operation der Kritik, diese Arbeit an sich selbst, kann ein Subjekt sich selbst transformieren und sich von den es konstituiert habenden Subjektivierungen emanzipieren; es kann im Subjekt dadurch also Unabhängigkeit und tatsächliche Eigenständigkeit gegenüber dem es konstituiert habenden Außen entstehen. Es entsteht Neues, das sich nicht auf Macht verrechnen lässt. Freiheit ist eine emergente Operationsebene gegenüber den das Subjekt konstituiert habenden Subjektivierungen innerpsychische Emergenz. Wie genau das Neue in die Welt kommt, lässt sich nicht vorhersagen; es lässt sich aber sagen, dass diese Art

Freiheitsbegriff der Kritik völlig anders gelagert ist als der ethische Freiheitsbegriff in Foucaults Spätwerk zur Antike, siehe Foucault, Michel: Der Gebrauch der Lüste. Sexualität und Wahrheit 2. Übers. von Ulrich Raulff und Walter Seitter. Frankfurt am Main: Suhrkamp 1989; ders.: Die Sorge um sich. Sexualität und Wahrheit 3. Übers. von Ulrich Raulff und Walter Seitter. Frankfurt am Main: Suhrkamp 1989; ders.: „Die Ethik der Sorge um sich als Praxis der Freiheit. Gespräch mit Helmut Becker, Raul Fornet-Betancourt und Alfred Gomez-Müller, 20.01.1984“, in: ders.: Schriften in vier Bänden. Dits et Ecrits - Band IV. 1980-1988. Hg. von Daniel Defert und François Ewald und übers. von Michael Bischoff. Frankfurt am Main: Suhrkamp 2005, 875-902 und dazu Schubert, Karsten: „Die christlichen Wurzeln der Kritik. Wie Foucaults Analysen der Kirchenväter neues Licht auf die Debatte um Macht und Freiheit werfen. Rezension von Michel Foucault: Die Geständnisse des Fleisches. Sexualität und Wahrheit 4“, in: Zeitschrift für philosophische Literatur 7 (2), 2019, 60-71.; Schubert: Freiheit als Kritik. 
innerpsychischer Emergenz durch freiheitliche Subjektivierungen wahrscheinlicher ist.

Als voraussetzungsreiche Fähigkeit kann Freiheit als Kritik nicht vorgängig im Subjekt angenommen werden. Vielmehr legt subjektivierungstheoretisches Denken es nahe, Freiheit als Kritik als ein Resultat von Subjektivierung aufzufassen - denn die Eigenschaften der Subjekte sind von Macht im Prozess der Subjektivierung instantiiert. Als paradigmatischen Fall einer solchen freiheitlichen Subjektivierung kann die genealogische Kritik gelten, die ihre Leser_innen dazu anregt, ihre eigene Subjektivierung kritisch zu reflektieren.

Doch mit dieser Feststellung der generellen Möglichkeit von Freiheit (durch Gesellschaftskritik) ist das Freiheitsproblem der Subjektivierung noch nicht gelöst, sondern nur verschoben. Dass Freiheit als Kritik generell möglich ist, ist zwar richtig, aber als Ergebnis unbefriedigend. Denn die generelle Möglichkeit von Freiheit kann nicht als Lösung dieses Problems gelten, in dessen Zentrum die begründete Befürchtung steht, dass wir viel unfreier sind, als wir denken. Wenn man sich mit der Aussage „Freiheit ist möglich“ zufrieden gibt, muss man dafür die Hermeneutik des Verdachts suspendieren - was widersprüchlich ist, war sie doch der Stein des Anstoßes. Kurz: Man verliert das Problem als sozialphilosophisches Problem aus den Augen, wenn man sich mit Aussagen in der Modalität der allgemeinen Möglichkeit zufrieden gibt, weil sie keine adäquate Antwort auf die begründete Befürchtung der weitgehenden Vermachtung sind. Die allgemeine Möglichkeit so festzustellen, ist zu unspezifisch. Damit ist zwar klar, dass freiheitliche Subjektivierung als Gesellschaftskritik immer möglich ist, aber damit ist noch keine weitere Aussage dazu gemacht, unter welchen Bedingungen sie wahrscheinlich ist.

Eine adäquate Antwort kann in der Umstellung der Modalitäten liegen: nicht allgemeine Aussagen der Möglichkeit, sondern Unterscheidungen verschiedener Wahrscheinlichkeiten sind sinnvoll. Entsprechend kann die Identifikation von solchen sozialen Situationen, in denen freiheitliche Subjektivierung wahrscheinlicher ist, als Lösung des Freiheitsproblems der Subjektivierung gelten. Es geht also darum, modal robuste Praxen bezüglich der freiheitlichen Subjektivierung zu identifizieren, also solche, die unter verschiedenen Umständen mit hoher Wahrscheinlichkeit freiheitlich subjektivieren. Solche modal robusten Subjektivierungspraxen können auch als Subjektivierungsregime bezeichnet werden. Die Notwendigkeit der Identifikation freiheitlicher Subjektivierungsregime leitet sich auch aus der beschriebenen langfristigen Wirkweise von Subjektivierung ab: Nur wenn die freiheitlich subjektivierende 
Gesellschaftskritik wiederholt und langfristig wirkt, kann davon ausgegangen werden, dass Subjekte die Fähigkeit zur Freiheit als Kritik ausbilden. Schon deshalb wäre es nicht genug für die Bestimmung von Freiheit, festzustellen, dass freiheitliche Subjektivierung immer möglich ist, weil dies nicht ausschließt, dass sie nur gelegentlich und zufällig auftaucht. Dieses „Argument der modalen Robustheit“ ist der entscheidende Schritt meiner Argumentation über die existierende Literatur zu Foucault hinaus. ${ }^{7}$ Aus ihm leitet sich die Notwendigkeit ab, das Problem der Freiheit in eine Frage nach der Institutionalisierung von Freiheit zu transformieren.

Ein naheliegendes Argument gegen diesen Gedanken besteht darin zu zeigen, dass Freiheit nicht in den oft repressiven Subjektivierungsregimen von politischen Institutionen, sondern in denjenigen von Protest- und Subkulturen verortet und weiter untersucht werden sollte. So kann bspw. queere Kritik an Heteronormativität und die von ihr entworfenen gegenhegemonialen Identifikationsangebote, die ,Normalität‘ subversiv umschreiben, als Paradebeispiel für freiheitliche Subjektivierung untersucht werden, und viele Foucauldianer_innen, wie beispielsweise Amy Allen $^{8}$ argumentieren für eine solche Verortung von Freiheit in Protestkultur.

Doch eine solche Verortung von Freiheit in einem spezifischen politischen Projekt wird dem prozeduralistischen und konflikthaften Charakter von Freiheit nicht gerecht. Wenn Freiheit als ein bestimmtes ethisch-politisches Projekt festgeschrieben wird, dann verliert sie ihren begrifflichen Kern der immer weitergehenden Hyperreflexion und es wird suggeriert, dass die eine Art von ethisch-politischer Subjektivierung, die von der ethischen-politischen Freiheitstheorie als gut ausgezeichnet wurde, unumstritten sei. Dies ist jedoch ein unzulässiges Argument in einer postfundamentalistischen Argumentation. Darüber hinaus geht die Hermeneutik des Verdachts, die der Freiheit als Kritik zugrunde liegt, davon aus, dass jede partikulare Politik der Freiheit leicht in Repression umschlagen kann. Deshalb ist es sinnvoll, Freiheit nicht nur in Protestbewegungen $\mathrm{zu}$ verorten, sondern die Fragestellung in eine demokratietheoretische zu transformieren, in der mit dem Problem der potentiell paternalistischen Normierungsansprüche von ethisch-politischen Projekten, also der Umstrittenheit von Freiheit, umgegangen werden kann. Dieser Argumentationsschritt geht endgültig über den Foucault'schen

$7 \quad$ Siehe meine detaillierte Rekonstruktion und Kritik der Debatte um Freiheit bei Foucault in Schubert: Freiheit als Kritik.

8 Allen: The Politics of Our Selves. 
Rahmen hinaus und führt den Begriff der Freiheit als Kritik (zurück) in die politische Theorie.

Dieses erste Argument für die Verortung von Freiheit in demokratischen Institutionen ist noch aus der formalen Logik von Freiheit als Kritik abgeleitet, und ihm könnte widersprochen werden, indem man die Protestbewegungen so reflexiv konzipiert, dass sie gegen das Umschlagen in Repression gewappnet sind, während man gleichzeitig argumentiert, dass politische Institutionen grundsätzlich repressiv seien - von Rancière werden sie in diesem Sinn „Polizei“ genannt. ${ }^{9}$ Wenn man den Freiheitsbegriff aber - wie Amy Allen ${ }^{10}$ dies tut - in einer immanenten Kritik als normativen Wert der Spätmoderne rekonstruiert und damit das Ziel begründet, Freiheit für alle, gesellschaftsweit zu verwirklichen, dann reicht der Fokus auf die (eigene) politische Protestbewegung nicht aus, sondern das allgemeine Institutionendesign der Gesellschaft rückt notwendig in den Fokus.

Zusammengefasst: Kombiniert man diesen Universalisierungsanspruch, der im normativen Freiheitsbegriff enthalten ist mit den Anforderungen an modale Robustheit und der Bestimmung von Freiheit als Kritik als ein Formprinzip der ständigen kritischen Reflexion gegen (politische) Normierung, folgt daraus, dass die kritische Reflexion dauerhaft und gesellschaftsweit angewendet werden sollte (und nicht nur in linken Bewegungen), weil nur so Freiheit für alle wahrscheinlich ist. Damit kann die Gefahr, dass die Einrichtung eines solchen Freiheitsregimes selbst repressive Effekte hat, nicht ausgeschlossen, sondern nur angemessenen prozessiert werden - durch die ständige Selbstverdächtigung der Hermeneutik des Verdachts.

Um die Unterscheidung des Liberalismus zwischen Moral und Ethik zu bemühen, sollte Freiheit auf der Ebene der Moral angesiedelt werden, also im allgemeinen politischen Institutionendesign der Gesellschaft verankert werden. Freiheit als Kritik hat hier die Funktion, in zwei Richtungen zu wirken: Einerseits fordert sie selbstreflexive, selbstkritische und änderungsoffene politische Institutionen, denn die Hermeneutik des Verdachts ist besonders wachsam gegenüber den Ansprüchen auf politische Universalisierung und den damit einhergehenden politischen Normierungen. Andererseits fordert sie die Einrichtung solcher Subjektivierungsregime, die zum Ziel haben, Freiheit als Kritik in allen Subjekten zu instantiieren. Freiheit als Kritik soll so auch auf alle ethisch- 
politischen Projekte wirken und ihren Mitgliedern die Fähigkeit geben, sich auch kritisch zu diesen ethisch-politischen Subjektivierungsregimen zu verhalten.

Der immer auch arbiträren ethisch-politischen Festsetzung von Freiheit kann nur durch eine weitere kritische Prozessierung begegnet werden. Der Anlass und die Energie zu dieser weiteren Prozessierung kommt dabei von den einzelnen Individuen, die sich transformieren und dabei auch die sie subjektivierenden Institutionen kritisieren und ändern. Dass sie dies tun, ist wahrscheinlicher, wenn sie freiheitlich subjektiviert wurden; die Dynamik der Institutionen kann aus der Perspektive der Freiheit als Kritik also durch die Institutionen selbst erzeugt werden. Und dennoch - das ist hier das entscheidende, weshalb ich diese Lesart auch als Foucault'schen Liberalismus bezeichne - bleibt die Kritik Sache der Individuen.

Soweit die Herleitung des Ansatzes einer Institutionentheorie aus dem Freiheitsproblem bei Foucault. Die Problematisierung von Institutionen im vorliegenden Band fragt quasi aus der umgekehrten Perspektive: Sie fängt mit der Fragestellung der politischen Theorie nach dem Verhältnis von Instituierung und Institution, Umbruch und Verstetigung an. Und für diese Fragerichtung ergeben sich folgende Konsequenzen aus dem bis hier Entwickelten.

Es gibt subjektive Voraussetzungen für Veränderung und Umbruch: kritische Subjektivitäten. Diese werden von der politischen Theorie, auch der postmarxistischen, kaum mitgedacht. Denn diese subjektiven Voraussetzungen von Politik können wiederum nur durch ein spezifisches Institutionengefüge hergestellt werden und sind damit im Rahmen der postmarxistischen Werdensphilosophie nicht ohne Weiteres bestimmbar. Implizit ist in dieser Feststellung eine Absage an eine Politik des Werdens enthalten, wenn sie radikal verstanden wird - als grundsätzlichen Umbruch, als Revolution oder als Exodus. Wenn die Menschen überhaupt erst im Immanenzraum der gegenwärtigen Institutionen zu kritischen politischen Subjekten werden, kann die radikale Transzendenz des Gegenwärtigen nicht die Ausrichtung der politischen Theorie und Praxis sein. Das bedeutet aber gerade nicht, dass Alterität, Singularität und Partikularität als einzuebnende und zu normalisierende Störungen aus dem Zentrum der Politik verdrängt werden. Im Gegenteil, sie bilden den normativen Kern der nach Foucaults Freiheitsbegriff skizzierten pluralistischradikaldemokratischen Institutionen, die dadurch das Werden erst ermöglichen. 


\section{Eine Kritik an Menkes Rechtskritik}

In diesem zweiten Teil konkretisiere ich die Theorie der Freiheit und der Institutionalisierung des Werdens, indem ich ihren Beitrag zur aktueller postmarxistischen Rechtskritik aufzeige. Dafür untersuche ich, wie der nach Foucault entwickelte Freiheitsbegriff der Kritik zentrale Aporien in Christoph Menkes Kritik der Rechte. ${ }^{11}$ aufheben kann. Mit Freiheit als Kritik ist es möglich, auf den Begriff zu bringen, worauf Menkes Projekt abzielt, aber wegen der Aporien nicht realisieren kann: die Spannung zwischen Liberalismus und Republikanismus, zwischen Recht und Politik aufzuheben und eine Kritik der liberalen Rechte zu denken, die die innere Unfreiheit der Subjekte und ihre Abhängigkeit vom Recht kritisiert und dennoch nicht paternalistisch ist. Meine vom Foucault'schen Freiheitsbegriff der Kritik informierte Auseinandersetzung mit Menke stellt dabei insbesondere auf zwei Punke ab: Erstens die Gefahr des Paternalismus, die mit einer politischen Kritik von Subjektivität einhergeht. Genau für dieses Problem bietet der Ansatz der Freiheit als Kritik eine Lösung. Und zweitens, die institutionellen und rechtlichen Bedingungen von kritisch-politischer Subjektivität zu problematisieren, die bei Menke unhinterfragt vorausgesetzt werden. Denn Menke denkt Subjektivierung rein repressiv und kann sie deshalb nicht als Bedingung der Möglicheit von Politik konzipieren. Menkes Projekt ist eine radikale Kritik an Gesellschaften, die auf der Vorstellung subjektiver Rechte basieren. Genauer unternimmt Menke eine Kritik der Subjektivierung durch bürgerlich-liberale subjektive Rechte, also der Art und Weise, wie Subjekte durch liberale Rechte konstituiert werden und wie sich das aus liberale Gesellschaften auswirkt. Damit ist Menkes Kritik wesentlich grundsätzlicher von Foucault bestimmt als die nur einen kleinen Teil des Buches einnehmende explizite Auseinandersetzung mit Foucault vermuten lässt.

Auf subjektiven Rechten beruhen nach Menke moderne Gesellschaften, die die spezifische liberale Form der subjektiven Rechte erfunden haben. Während das antike und römische Recht die Gerechtigkeit, die vernünftigen Ordnung und das ethische Leben ausdrücken sollte, wird in der Moderne der Wille von Individuen zur Grundlage des Rechts. ${ }^{12}$ Der individuelle Wille wird als vorgesellschaftliche Naturgewalt und Freiheit verstanden, und im modernen Recht geht es um die Anerkennung und den Schutz dieser 
Natur. Daher basiert das moderne Recht auf subjektiven Rechten, und subjektive Rechte sind „(rechtliche) Ansprüche auf (natürliche) Ansprüche, die rechtliche Ermächtigung von natürlicher Macht.“13 Die Form der modernen subjektiven Rechte revolutioniert das Verhältnis von Norm und Natur völlig und stellt die Natur in den Vordergrund. ${ }^{14}$ Die natürlichen Rechte sind jetzt primär dem Recht untergeordnet; das „objektive Recht“ wird eingerichtet, um das ,subjektive Recht“ zu sichern. ${ }^{15}$

Dieses moderne Primat der Rechte hat grundlegende Auswirkungen auf die Gesellschaft, da es das Selbstverständnis des Menschen verändert - und es bestimmt, wer wir heute sind. Während die Naturrechtstradition sagt, daß das autonome Subjekt der Grund für die subjektiven Rechte ist, dreht Menke die Verursachungsbeziehung kritisch um: Wir haben nicht die liberale Ordnung, um unsere Freiheit und unseren natürlichen, individuellen Willen zu sichern, wie der klassische Liberalismus sagt, sondern wir sehen uns selbst erst aufgrund der liberalen Rechtsordnung als individuelle Subjekte. Das ist die Subjektivierung durch das Recht. Das autonome, individuelle und egoistische Subjekt der subjektiven Rechte (des bürgerlichen Liberalismus) ist das Produkt des Primats der Rechte im liberalen Recht. Indem es den individuellen Willen vor politischem Einfluss schützt, schafft es Egoismus und führt so zu einer amoralischen Gesellschaft ohne normative Gründe. Der private Wille des Subjekts ist vor Politik und Ethik geschützt. Weil sich der Wille nicht rechtfertigen muss, wird die Politik als Rechtfertigungsprozess blockiert; die bürgerliche Gesellschaft ist eine entpolitisierte und entsittlichte Gesellschaft. ${ }^{16}$

Dabei hat das moderne Recht nach Menke die historische Chance auf Reflexivität und Emanzipation - die es aber verspielt, weil es nur in seiner bürgerlichen Verfallsform realisiert wird, die Menschen so subjektiviert, dass sie ihren Willen als natürlich, eigen und von Gesellschaft und Politik unabhängig denken. Grundsätzlich ist das moderne Recht nach Menke reflexiv, weil es zum ersten Mal die Lücke zwischen Recht und Nichtrecht

13 Ebd., 56.

14 Ebd., 39.

15 Der Unterschied zwischen ,das Recht‘ und ,einem Recht‘, zwischen ,Recht‘ als Rechtsordnung und ,Rechten“ als individuelle Ansprüche, ist eine moderne Erfindung und kann auf Wilhelm von Ockham (ca. 1288-1347) zurückgeführt werden, wird bereits von Thomas Hobbes (1588-1679) als selbstverständlich vorausgesetzt. Der Begriff „subjektives Recht“ im Unterschied zu „objektives Recht“ wird verwendet, um diese Unterscheidung explizit zu machen, da beide Begriffe mit dem gleichen Wort „Recht“ bezeichnet werden (ebd., 19-21.). 
reflektiert. Im traditionellen Recht wurde die Kluft zwischen Recht und Nicht-Recht in einer höheren Ordnung und Einheit (Natur im antiken Recht, Vernunft im römischen Recht) versöhnt, also durch eine fundamentalistische Konzeption, die die Willkür des Rechts unsichtbar macht. Die Innovation des modernen Rechts besteht darin, dass es seine eigene Lücke deutlich macht und reflektiert. Die Reflexion der paradoxen Struktur zwischen Natur und Recht ist der Weg des modernen Rechts aus dem traditionalistischen Fundamentalismus. Mit anderen Worten: Das moderne Recht weiß, dass es keine gegebene Ordnung der Legalität und Moral gibt (wie im römischen und antiken Recht), und eröffnet daher die Möglichkeit der Reflexion dieser Unbegründetheit. Reflexives modernes Recht sieht sich in Wechselwirkung mit dem Nicht-Recht, es kann konzeptualisieren, dass es selbst negative Auswirkungen auf die Gesellschaft haben kann (beispielsweise, dass eine bestimte liberale Rechtsund Eigentumsordnung zu Verarmung führt), und kann auf den Protest gegen eine solche Auswirkungen reagieren. In diesem Rahmen werden komplexe rechtliche Mechanismen der Prozeduralisierung und demokratischen Kontrolle etabliert. ${ }^{17}$

Menkes Kritik am modernen Recht ist jedoch, dass seine Reflexivität nur begrifflich oder potenziell ist; die einzige realisierte Form des modernen Rechts ist das bürgerliche Recht, das diese Reflexivität blockiert. Das bürgerliche Recht blockiert die Reflexivität, indem es die Natur durch das Recht essentialisiert, was das Gegenteil eines Verständnisses des Verhältnisses von Recht und Natur als Dialektik ist. Anstatt darüber zu reflektieren, wie es Subjekte konstituiert - die Prozesse der Subjektivierung durch das Recht -, arbeitet die Subjektivierung des Rechts durch die Vernachlässigung seiner eigenen Effekte, indem sie Subjekte mit einem natürlichen, vorgesellschaftlichen und unveränderlichen Willen und Interesse konzeptualisiert. Menke nennt dies „Empirismus und Positivismus“ und in Anlehnung an Wilfrid Sellars den „Mythos des Gegebenen“"18. Das Grundproblem dieses Positivismus besteht nach Menke darin, dass das Subjekt, das das Gesetz mit dem Ziel des Schutzes seines

17 Vgl. ebd., 135-155.; Luhmann, Niklas: „Verfassung als evolutionäre Errungenschaft“, in: Rechtshistorisches Journal 9, 1990, 176-220 ist eine Erklärung für diesen Prozess: Durch die Verfassung kann das Recht strukturell mit der Politik gekoppelt werden, was die Institutionalisierung des Rechtswandels und damit die Fähigkeit zur Reaktion auf politische Kämpfe und gesellschaftliche Veränderungen ermöglicht. 
Willens und seiner Interessen schafft, ein amoralisches Subjekt ohne normative Gründe ist, ebenso wie die liberal-bürgerliche Gesellschaft. ${ }^{19}$ Die ontologische Neuerung (und das Scheitern) des bürgerlichen Rechts besteht darin, eine radikal egoistische und asoziale Gesellschaft zu schaffen, die ausschließlich auf dem privaten Willen der Individuen beruht. Die Neuerung besteht darin, dass der individuelle Wille nicht, wie im traditionellen Recht, gegen eine politische Normativität geprüft wird. Der private Wille des Subjekts wird vor Politik und Ethik geschützt. Weil der Wille sich nicht rechtfertigen muss, wird Politik als Rechtfertigungsprozess blockiert; die bürgerliche Gesellschaft ist eine entpolitisierte Gesellschaft. ${ }^{20}$ Die Eigentumsrechte sind die Hauptform der subjektiven Rechte, die sich so entpolitisieren; es sind Rechte zum Ausschluss anderer, die nicht nur gegen andere Individuen, sondern gegen Ethik und Vernunft insgesamt durchgesetzt werden. Das Wesen des Eigentums besteht darin, frei von normativen und politischen Forderungen bezüglich der Nutzung des Eigentums zu sein. ${ }^{21}$ Die bürgerliche Entpolitisierung und „Entsittlichung“22 arbeitet durch die Etablierung der Differenz zwischen der bürgerlichen Gesellschaft (als vor-politischer) und der Politik und durch die Beschränkung der Politik auf die Sicherung der subjektiven Rechte. ${ }^{23}$ Politik als gemeinsame Entscheidungsfindung über kommunale Belage wird dadurch unmöglich gemacht, weil kein politischer Wille gegen den privaten Willen entwickelt werden kann.

Das letzte Kapitel von Menkes Monographie widmet sich der Entwicklung eines neuen Rechtsbegriffs, der es ermöglichen soll, die bürgerlich-liberale Pathologie der Entpolitisierung zu überwinden. Dieses Konzept ist ein repolitisiertes „neues Recht“ mit den Gegenrechten, das auf

19 Vgl. ebd., 202-207, 251-252.

20 Vgl. ebd., 358.

21 Vgl. ebd., 209-214. Auch soziale Rechte, die normalerweise gegen liberale Rechte gestellt werden, basieren auf dem Paradigma der subjektiven Rechte nach Menke, da sie die Fähigkeit des Individuums garantieren sollen, seinem freien Willen zu folgen (ebd., 215-225). Liberale Forderungen nach umfassenden Eigentumsrechten und die Reaktion der „sozialistischen“ Forderungen nach sozialen Rechten sind der bürgerliche Kreis der Intensivierung der Regierung. Liberale Rechte erzeugen soziale Probleme, die das Bedürfnis nach sozialen Rechten erzeugen, die Paternalismus erzeugen, die das Bedürfnis nach liberalen Rechten erzeugen, und der Kreis beginnt von neuem. Es ist jedoch nicht klar, warum dieser Kreis eine Intensivierung darstellt (ebd., 243-248, 281-294.).

22 Ebd., 317.

23 Vgl. ebd., 318, 325f. 
Partizipation basiert. Das neue Recht soll eine Repolitisierung des individuellen Willens und demokratische Teilhabe ermöglichen und gleichzeitig die Fallstricke des politischen Paternalismus vermeiden. Menke arbeitet das Problem der Bevormundung unter dem Stichwort „Kommunismus“ durch, womit er eine politische Selbstbestimmung der Gemeinschaft meint, die total ist und keinen Raum für individuelle Passivität gibt - im Kommunismus gibt es nur Politik. Hier ist also ein offensichtliches - und eher klassisches - Problem angesprochen: Totale demokratische Kontrolle und totale Politisierung kann zu Bevormundung führen. Individuen und Minderheiten könnten gezwungen werden, sich an die Gesellschaft anzupassen. Im Gegensatz zum (totaldemokratischen und paternalistischen) Kommunismus soll das neue Recht politische Selbstbestimmung ermöglichen und gleichzeitig Passivität zulassen, die durch Gegenrechte gesichert ist. Der Unterschied zu liberalen subjektiven Rechten besteht darin, dass die Gegenrechte keine Rechte auf einen naturalisierten egoistischen Willen sind, der vor politischen Einfluss geschützt sein soll, sondern auf die Berücksichtigung von Sinnlichkeit und Passivität im Prozess der politischen Beurteilung. Da der als vorpolitisch imaginierte private Wille und das private Interesse nun nicht mehr vor politischem Einfluss geschützt werden müssen, eröffnet und unterstützt das neue Recht die Möglichkeit eines echten politischen Wandels, der politischen Steuerung des Sozialen ohne vorgegebene (ideologischbürgerliche) Grenzen durch den privaten Willen.

Ich möchte darauf hinweisen, dass die zentrale Erkenntnis von Menkes Theorie die Verbindung von Rechtskritik mit der Theorie der Subjektivierung ist. Subjektivität und der individuelle Wille sind nicht vorsozial, sondern das Ergebnis der Subjektivierung in einer bestimmten Gesellschaft - im modernen, bürgerlichen Liberalismus ist dies eine Subjektivierung auf der Grundlage subjektiver Rechte. Es handelt sich um Individuen, die fühlen und denken, dass es natürlich ist, ein Leben in bürgerlicher amoralischer Trennung zu führen, und die glauben, dass es Paternalismus ist, wenn die Politik ihr Privat- und Wirtschaftsleben stört. Dies ist eine Kritik am klassischen politischen Liberalismus und seinem negativen Konzept von Freiheit. Die Frage ist, ob dieses negatives Konzept von Freiheit und die Blindheit gegenüber der Subjektivierung überwunden werden kann, ohne den Albtraum des Liberalismus, eine paternalistische Gesellschaft, wahr zu machen.

Meine Kritik an Menke ist, dass ihm genau das nicht gelingt, weil die Gegenrechte keinen ausreichenden Schutz vor politischer Bevormundung bieten. Anders gesagt: Menkes Ziel ist es, den Unterschied in der 
politischen Theorie zwischen einer liberalen und republikanischen Position zu überwinden, wobei die liberale Position auf universellen Rechten des Einzelnen besteht und die republikanische Position die politische Selbstbestimmung der Gemeinschaft fokussiert. ${ }^{24}$ Aber entweder, einer starken Lesart folgend, führt das neue Recht $\mathrm{zu}$ einem radikalen Republikanismus, der den individuellen Willen durch totale Politisierung außer Kraft setzt. Oder - einer schwachen Lesart folgend - auch Menkes Gegenrechte bleiben letztlich strukturgleich mit den liberalen subjektiven Rechten, und er kann den liberalen Rahmen nicht verlassen.

1. Starke Lesart: Radikaler Republikanismus. Nach Menke besteht das Problem darin, dass durch die Form der subjektiven Rechte der individuelle private Wille geschaffen wird, der gegen politische und gesellschaftliche Kontrolle gesichert und unabhängig davon ist - subjektive Rechte schaffen „das positive Vermögen zur sittlichen Indifferenz“. ${ }^{25} \mathrm{Er}$ nennt das „Entsittlichung“. Dagegen plädiert er für eine politische Kontrolle, die konzeptionell nicht durch die Privatsphäre begrenzt ist. Dies ist ein gefährlicher Weg, da er das massive Problem der Bevormundung durch grenzenlose Politik übersieht. Viele politische Fortschritte von Minderheiten werden durch Rechte gesichert, indem die liberale Form der subjektiven Rechte genutzt wird, um Schutz vor repressiver und normalisierender Politik zu fordern. Zum Beispiel wurden wichtige Schritte für den Schutz der Menschenrechte auf sexualle Selbstbestimmung durch das Recht auf Privatsphäre des Internationalen Konvents über bürgerliche und politische Rechte in der Entscheidung Toonen vs. Australien im Jahr 1994 geprägt, die einvernehmlichen privaten schwulen Sex erlaubte. ${ }^{26}$ Aus einer queeren Perspektive erscheint Menkes Vision einer durch und durch

24 Ein Ziel, das er mit Jürgen Habermas teilt: ders.: „Drei normative Modelle der Demokratie“, in: ders.: Die Einbeziehung des Anderen. Studien zur politischen Theorie. Frankfurt am Main: Suhrkamp 1996, 277-292.; ders.: „Zur Legitimation durch Menschenrechte“, in: ders.: Die postnationale Konstellation. Politische Essays. Frankfurt am Main: Suhrkamp 1998, 170-192, ungeachtet der tiefen Unterschiede in den philosophischen Methoden und Lösungsvorschlägen. Menke: Kritik der Rechte, 253.

26 Vgl. Human Rights Committee "Toonen v. Australia, Communication No. 488/1992, 1994, 31.3.1994, URL:

<http://www1.umn.edu/humanrts/undocs/html/vws488.htm>, zur historischen Genese, zum aktuellen Stand und zu Problemen des internationalen Menschenrechtsrahmens hinsichtlich sexueller Orientierung und Geschlechtsidentität vgl. Schubert, Karsten: „Langer Weg zur sexuellen Selbstbestimmung. Der Schutz von LSBTI durch die Vereinten Nationen“, in: Vereinte Nationen 61 (5), 2013, 216-222. 
politischen Gesellschaft ohne gesichterte Privatbereiche als bedrohlich, weil hier einheitliche Normierung statt Diversität zu befürchten ist. Kurz: Aus einer Minderheitenperspektive sind subjektive Schutzrechte ein zentrales politisches Mittel und es wundert nicht, dass radikale und grundsätzliche Kritik nicht aus einer Analyse der politischen Kämpfe von Minderheiten heraus entwickelt wird, sondern durch eine abstrakte und universelle philosophische Analyse. ${ }^{27}$

Systematischer formuliert besteht das Problem des radikalen Republikanismus darin, dass er strukturell antipluralistisch ist, da er sich auf ein universalistisches Konzept der politischen Vernunft stützt und den politischen Konflikt ausblendet. Gegen Lefort, Gauchet, Rawls und implizit alle TheoretikerInnen, die über postfundamentales politisches Denken und die politische Differenz arbeiten, ${ }^{28}$ besteht Menke darauf, dass es in der erscheint Menkes Kritik leicht als eine ausgefeiltere Version der Critical Legal Studies (CLS), die eine radikale Rechtskritik aus marxistischer Perspektive bot Fitzpatrick, Peter/Hunt, Alan (Hg.): Critical legal studies. Oxford: Blackwell 1990; Boyle, James (Hg.): Critical legal studies. Aldershot: Dartmouth 1992 (The international library of essays in law and legal theory Schools 4). Critical legal studies wurde von der Critical Race Theory kritisiert, weil sie alle Fragen durch Klassenkampf und Ideologie erklärt (Menkes Äquivalent wäre „Form der subjektiven Rechte"), was Critical legal studies unfähig macht, die Bedeutung rechtlicher Kämpfe im Kampf gegen Rassismus anzuerkennen, vgl. Williams, Patricia J.: The Alchemy of Race and Rights. Diary of a Law Professor. Cambridge/Massachusetts: Harvard University Press 1991; Alexander-Floyd, Nikol G.: „Critical Race Black Feminism: A "Jurisprudence of Resistance" and the Transformation of the Academy“, in: Signs: Journal of Women in Culture and Society 35 (4), 2010, 810-820.

28 Siehe Lefort/Gauchet: „Über die Demokratie: Das Politische und die Instituierung des Gesellschaftlichen“; Rawls, John: Die Idee des politischen Liberalismus. Aufsätze 1978-1989. Hg. von Wilfired Hinsch. Frankfurt am Main: Suhrkamp 1992; ders.: Politischer Liberalismus. Übers. von Wilfried Hinsch. Frankfurt a. M.: Suhrkamp 1998; Laclau, Ernesto/Mouffe, Chantal: Hegemony and socialist strategy. Towards a radical democratic politics. 2. Auflage. London: Verso 2001; Mouffe, Chantal: On the political. London: Routledge 2008 (Thinking in action); Butler, Judith/Laclau, Ernesto/Žižek, Slavoj: Contingency, hegemony, universality. Contemporary dialogues on the Left. London: Verso 2000; Rancière: Das Unvernehmen; Marchart: „Politische Theorie als Erste Philosophie. Warum der ontologischen Differenz die politische Differenz zugrunde liegt“. 
Politik um (universalistische) Vernunft geht, die gegen (partikulare) Individualität gestellt wird. ${ }^{29}$

Die Vernunft ist nach Menke nicht nur eine weitere Partikularität, die es durch politische Hegemoniekämpfe geschafft hat, dominant zu werden, sondern die Kategorie der universalistischen politischen Vernunft existiert wirklich und steht gegen die Individualität. ${ }^{30}$ Dieser Idealismus ist das Gegenteil einer konfliktbasierten politischen Ontologie, wie sie der Debatte um das Politische, dem Postfundamentalismus und dem Foucault'schen Freiheitsbegriff der Kritik zugrunde liegt. Von universalistischer Vernunft auszugehen und den Pluralismus abzublenden ist die philosophische Basis dafür, das Problem der paternalistischen Normierung zweitrangig zu behandeln und die Politik gegenüber dem Recht total werden zu lassen. Wenn hingegen eine pluralistische und konflikthafte politische Ontologie mit einem perspektivitischen Vernunftbegriff der Ausgangspunkt ist, dann tritt das Problem, wie politische Steuerung funktioniert, ohne dabei paternalistisch zu normieren, in den Mittelpunkt. ${ }^{31}$ Genau mit diesem Problem beschäftigen sich radikale Demokratietheorien, wie der Ansatz der Freiheit als Kritik. ${ }^{32}$

29 Dazu Menke: „Der Gewinn von Allgemeinheit ist aber nichts anderes als der Prozeß der Vernunft. Also kann die besondere Individualität, die von der je besonderen politischen Entscheidung übermächtigt wird, nur die nichtvernünftige Individualität sein: die Individualität gegen die politische Vernunft“ (Menke: Kritik der Rechte, 361).

30 Die Leser_in mag an die Frage erinnert werden, wie liberal oder paternalistisch der Rousseau'sche Unterschied zwischen volonté general und volonté de tous $\mathrm{zu}$ lesen ist; Inston, Kevin: Rousseau and radical democracy. London: Continuum 2010 zeigt, wie man ihn mit dem Pluralismus der zeitgenössischen radikalen Demokratie kompatibel machen kann.

31 Hier liegt eine bemerkenswerte Verwandtschaft zwischen Isaiah Berlins und Foucaults Liberalismus. Auch Berlin analysiert den strukturellen Paternalismus Vernunftbehauptungen, durch die Intellektuelle sich anschicken, philosophisch das Gute und Richtige festzulegen und gleichzeitig mit einer Theorie der (Un)Freiheit erklären, wieso noch nicht alle diese Erkenntnis erlangt haben, um sie dann, auch unter Einsatz von Zwangsmittel, zu befreien, vgl. Berlin, Isaiah: „Two Concepts of Liberty“, in: ders.: Liberty. Hg. con Henry Hardy. Oxford: Oxford University Press 2002, 166-217. Foucault teilt diese Sorge und entwickelt seine genealogische Methode, um solchen Paternalismus $\mathrm{zu}$ vermeiden.

32 Siehe in diesem Zusammenhang auch meinen Vorschlag für eine radikale Politisierung auf der Grundlage einer partikularen politischen Ontologie und perspektivistischen Epistemologie in Schubert, Karsten: „,'Political Correctness“ als Sklavenmoral? Zur politischen Theorie der Privilegienkritik“, in: Leviathan 48 (1), 2020, [im Erscheinen]. 
2. Schwache Lesart: Gegenrechte als Schutzrechte. Da die radikalrepublikanische Lesart in Paternalismus führt, bespreche ich im Folgenden eine prima facie attraktivere schwächere Lesart. In deren Mittelpunkt steht Menkes Vorschlag, die Differenz zwischen Liberalismus und Republikanismus durch das neue Recht und die Gegenrechte aufzuheben. Die Gegenrechte sollen die Nichtteilnahme bei einer allgemein kommunistischen Konzeption von Politik, die Freiheit mit politischer Partizipation gleichsetzt, schützen und ermöglichen. Sie sollen also der paternalistischen Totalpolitisierung, die das Problem der starken radikalrepublikanischen Lesart ist, einen Riegel vorschieben. Es bleibt jedoch unklar, inwiefern sich die Gegenrechte wirklich systematisch von der liberalen Form der subjektiven Rechte unterscheiden. Während die bürgerlich-subjektiven Rechte Passivität (Sinnliches) als Recht essenzialisieren und gegen das Urteilen stellen, bejahen die Gegenrechte Passivität, Leiden und Sinnliches, indem sie sie zum Ausgangspunkt für politische Urteile machen. ${ }^{33}$ Was damit gemeint sein könnte, lässt sich am besten durch einen politischen Prozess der Entwicklung einer Minderheiten schützenden Politik erklären, z.B. die Entwicklung einer politischen Position (Urteilen), die Schwule nicht diskriminiert, indem sie das Leiden an vergangener Diskriminierung (Sinnliches) berücksichtigt. Die Einbeziehung des Sinnlichen in die Beurteilung soll das Sinnliche jedoch nicht essentialisieren, sondern in den politischen Prozess einbeziehen und es verhandeln. Das Problem ist erneut ein Oszillieren zwischen zwei möglichen Interpretationen: Wenn es keine begriffliche Begrenzung der Debatten über Sinnliches in der Politik gibt, sondern das politische Urteil die Definitionsmacht darüber hat, welche sinnlichen Eindrücke zu berücksichtigen und einzubeziehen sind und welche nicht, dann läuft die Position auf radikalen Republikanismus hinaus und bringt keine Hilfe im Kampf gegen seinen Paternalismus. So könnte in einer homophoben politischen Vernunft das Leiden an Homophobie als ein sinnlicher Eindruck beurteilt werden, der ein völlig unproblematischer Nebeneffekt einer guen politischen Erziehung zum ,richtigen' Lebenstil ist. Wenn es andererseits eine Grenze für die Politisierung des Sinnlichen gibt, dann unterscheidet sich die Position konzeptionell nicht vom bürgerlich-liberalen Recht, dessen Wesen nach Menke darin besteht, das Sinnliche vom politischen Urteil zu befreien. 
Diese Probleme werden noch komplizierter, wenn man bedenkt, dass nicht nur das Sinnliche das Urteilsvermögen beeinflusst, sondern auch umgekehrt, dass das Urteilsvermögen das Sinnliche beeinflusst. ${ }^{34}$ Eine weitere Frage, die Menke offen lässt, ist, wie genau der Unterschied zwischen Sinnlichem und Urteilsvermögen verstanden werden kann: In politischen Kämpfen vermischen sich typischerweise beide. Was ist z.B. der Status des religiösen Sinnlichen, das eindeutig nicht nur ein körperlicher Affekt ist, sondern eine ganze Weltanschauung voller Urteile. Ein weiteres Problem ist, dass der Aufbau des Konzepts der Gegenrechte auf dem Sinnlichen zu Argumenten auf der Grundlage eines essentialistischen Biologismus führen kann, z.B. über die „Natur“ von „Männern“ und „Frauen“ bezüglich der Verteilung von sozialen Verpflichtungen und Privilegien. Am Kampf um sexuelle Selbstbestimmung kann man das Problem von essentialisierenden Strategien gut nachvollziehen. Amerikanische Schwule behaupten, sie seien schwul geboren (biologistisch essentialistisch) um sich gegen die homophoben Anfeindungen zu wehren, Homosexualität sei ein unmoralischer Lebensstil und damit eine freie Wahl. Doch dieses „born-that-way“-Argument greift überhaupt nicht die Behauptung der Amoralität an; damit ist sie defensiv und schützt darüber hinaus nicht vor biotechnologischen Veränderungen der sexuellen Orientierung, wenn diese verfügbar werden. ${ }^{35}$

Systematisch läuft dies auf ein generelles Problem hinaus, das Sinnliche als Grundlage der Politik zu konzipieren, hinaus: Es ist antipluralistisch. Das Sinnliche in der Politik zu berücksichtigen ist viel schwächer als eine Pluralität (amoralischer) individueller Wünsche zuzulassen. Das Sinnliche wird in die universalistische Vernunft eingearbeitet und gegen sie geprüft; was aber notwendig ist, ist ein Schutz einer Pluralität von Gründen, die im

34 Vgl. Nussbaum, Martha C. „Konstruktionen der Liebe, des Begehrens und der Fürsorge“, in: dies.: Konstruktionen der Liebe, des Begehrens und der Fürsorge. Drei philosophische Aufsätze. Übers. von Joachim Schulte. Stuttgart: Reclam 2002, ___ hier: 163.

35 Vgl. Stein, Edward: „Born That Way? Not A Choice?: Problems with Biological and Psychological Arguments for Gay Rights“, in: Cardozo Legal Studies Research Paper No. 223 2008; Stein, Edward: „Sexual Orientations, Rights, and the Body: Immutability, Essentialism, and Nativism“, in: social research 78 (2), 2011, 633-658, hier: 633. 
Liberalismus durch die Differenz zwischen Moral (universalistisch) und Ethik (partikular) konzeptualisiert wird. ${ }^{36}$

Im Ergebnis: Radikaler Republikanismus ist problematisch, und die Gegenrechte können einen Rückfall in den radikalen Republikanismus nur verhindern, wenn sie konzeptionell wie subjektive Rechte sind, die nicht nur das Sinnliche ins politische Urteilen bringen, sondern auch willkürliches Urteilen (des individuellen Willens und der Ethik) vor politischem Zugriff schützen. Menke kann die Spannung zwischen Liberalismus und Republikanismus, zwischen Recht und Politik, zwischen dem Sinnlichen und dem Urteil durch sein Konzept des Neuen Rechts nicht auflösen.

\section{Schluss: Recht mit Foucault gedacht}

Die postmarxistische politische Philosophie bevorzugt analytisch und normativ die Veränderung gegenüber der Verstetigung. Deshalb bezeichne ich sie als eine Philosophie des Werdens. Problematisch ist dies, weil in diesem Paradigma des Werdens keine realistische Analyse und normativdifferenzierende Bewertung von politischen Institutionen, insbesondere des Rechts, möglich ist. Es kann nicht untersucht werden, in welchem Verhältnis Emanzipation, politische Veränderung und rechtliche Institutionen stehen. Während dieses Problem in der Debatte um ,das Politische“ insbesondere als eine Leerstelle, also der Abwesenheit der Frage nach ,guter Ordnung', auftritt, legt Menke eine postmarxistische Rechtstheorie vor, in deren Zentrum die Frage nach dem Verhältnis von rechtlicher Verfestigung und politischer Veränderung steht. Doch Menke löst dieses Verhältnis einseitig zur politischen Veränderung hin auf. Damit liefert auch er keine Theorie der Verstetigung der Veränderung, die nötig wäre, um die postmarxistische Werdensphilosophie zu transformieren.

Die Analyse und Kritik von Menkes Text hat zu fundamentalen Ergebnissen geführt, die dabei helfen, eine solche Theorie der Verstetigung

Vgl. Habermas, Jürgen: Faktizität und Geltung. Beiträge zur Diskurstheorie des Rechts und des demokratischen Rechtsstaats. 2. Auflage. Frankfurt am Main: Suhrkamp 1992; Forst, Rainer: „Ethik und Moral“, in: Wingert, Lutz/Günther, Klaus (Hg.): Die Öffentlichkeit der Vernunft und die Vernunft der Öffentlichkeit. Festschrift für Jürgen Habermas 2001. Frankfurt am Main: Suhrkamp 2001, 344-371.; Rosenfeld, Michel: Law, justice, democracy, and the clash of cultures. A pluralist account. Cambridge/New York: Cambridge University Press 2011. 
der Veränderung zu etablieren. Ich habe gezeigt, dass das zentrale Verdienst von Menkes Rechtskritik die Analyse der Subjektivierung durch bürgerlichliberales Recht ist, die zu liberaler Entpolitisierung führt, und dass das zentrale Problem sein Antipluralismus ist. Damit ist die systematische Frage aufgeworfen, wie man das Problem der Subjektivierung angehen kann, ohne in Antipluralismus zu verfallen? Mit anderen Worten: Kann es einen pluralistischen (und das heißt liberalen) Weg geben, um das Problem der Subjektivierung anzugehen?

Und dabei hilft der Foucault'sche Freiheitsbegriff, mit dem die unproduktive Alternative von klassischem Liberalismus und radikalem Republikanismus überwunden werden kann. Denn diese Alternative, in der Menke feststeckt, ist bedingt durch die Beschränkung auf die zwei zugehörigen Freiheitsbegriffe: einerseits der klassisch negative Freiheitsbegriff, den Menke am Kern der subjektiven Rechte sieht, und dessen entpolitisierende Wirkung er so treffend herausarbeitet. Und andererseits der positive Freiheitsbegriff, der in einer republikanischholistischen Sozialontologie nach Arendt und Hegel begründet ist, wonach Freiheit mit Partizipation an der gemeinschaftlichen Selbstregierung gleichgesetzt ist. Mit dem Freiheitsbegriff der Kritik steht nun ein Begriff in Äquidistanz zum negativen und positiven Freiheitsbegriff zur Verfügung. Er leistet eine genauso radikale Kritik des Begriffs der subjektiven Rechte, wie Menke sie anführt, nämlich eine Kritik ihrer bürgerlichenessentialisierenden Subjektivierung. Und dies, ohne die üblichen Kosten dafür zu produzieren: ungebremsten politischen Paternalismus. Denn während die Kritik von Subjektivierungen, angeleitet durch freiheitliche Subjektivierungsregime, immer einen partikularen politischen Inhalt hat und damit potentiell paternalistisch ist, ist Freiheit als Kritik möglichst formal darauf ausgerichtet, die einzelnen Individuen mit der Fähigkeit der Subjektivierungskritik auszustatten. Der Kern dieser Subjektvierung ist die Ermächtigung der Individuen zur immer weitergehenden Kritik von subjektivierenden Regimen, womit das Subjektivierungsregime der Kritik die am wenigsten paternalistische Art der Subjektivierungskritik ist, die möglich ist. ${ }^{37}$ 
Dieser Freiheitsbegriff spricht außerdem dafür, dass subjektive Rechte, trotz ihrer teilweise pathologisch-essentialisierenden Funktion, ein notwendiges Element von kritischen Subjektivierungsregimen sind. Die reale Praxis der Rechtseinforderung führt zu kritischer Subjektivität - das ist die positive, freiheitliche Seite von rechtlicher Subjektivierung, auf die, wenn auch mit anderem Vokabular, Jay Bernstein hingewiesen hat. ${ }^{38}$ Für die Frage nach den Institutionen bedeutet dies: Recht ist nicht nur das Sozialsystem der Regelverstetigung und Institutionalisierung, sondern durch die Wirkung von rechtlicher Subjektivierung auf die Individuen auch ein zentraler Treiber gesellschaftliche Veränderung. Damit ist im Recht, genauer in freiheitlicher Subjektivierung durch subjektive Rechte, eine zentrale Sphäre der Verfestigung von Veränderung, der Verstetigung des Werdens identifiziert.

Die Rede vom Neuen Recht und den Gegenrechten aufzugeben, weil sie in den Aporien der alten (negativen und positiven) Freiheitsbegriffe gefangen ist und anstelle dessen eine Rechtstheorie auf dem Begriff der Freiheit als Kritik aufzubauen, eröffnet neue Möglichkeiten. Es erlaubt, weniger radikal und realistischer zu fragen, wo die Politik durch die Sprache der Rechte und ihre liberale Subjektivierung problematisch blockiert wird, und welche subjektiven Rechte dennoch hilfreich sind und sogar, als Teil eines freiheitlichen Subjektivierungsregimes, bei der Kritik der Subjektivierung helfen können.

Eine Theorie des Rechts auf Grundlage des Foucault'sche Freiheitsbegriffs der Kritik zu entwickeln kann dabei helfen, die einseitige Fokussierung auf das Werden und politische Veränderung in der postmarxistischen Gesellschaftstheorie zu überwinden, ohne in alte Essentialismen zurückzufallen. So kann die Bedeutung von verstetigten rechtlichen Strukturen für den politischen Prozess analysiert werden. Erst durch freiheitliche Subjektivierung im Recht gibt haben Individuen die Möglichkeit, politische Veränderungsprozesse zu gestalten. Die zentrale Innovation dabei ist erstens, dass ein wechselseitiges Verhältnis zwischen Recht und Politik zum ersten Mal aus der postmarxistischen

politischen Theorie. Baden-Baden: Nomos 2020 [im Erscheinen], auch erschienen als ders.: „Der letzte Universalismus. Kontingenz, Konflikt und normative Demokratietheorie.“ Schwerpunktbeitrag 2017, URL:

$<$ https://philosophie-indebate.de/2995/schwerpunktbeitrag-der-letzteuniversalismus-kontingenz-konflikt-und-normative-demokratietheorie/>.

38 Vgl. Bernstein, Jay: „Rights“, in: Political Concepts. A Critical Lexicon 2012, URL:

$<$ https://www.politicalconcepts.org/rights-bernstein/>. 
Institutionalisierung der Freiheit im Recht

Theorietradition entwickelt wird. In diesem neuen Begriff des Rechts können zweitens die zentralen Topoi des postmarxistischen Denkens, die bis jetzt in einem Widerspruch standen - Politisierung, Subjektivierung und Anti-Paternalismus - endlich kohärent zusammen gedacht werden. 


\section{Literaturverzeichnis}

Alexander-Floyd, Nikol G.: „Critical Race Black Feminism: A 'Jurisprudence of Resistance' and the Transformation of the Academy“, in: Signs. Journal of Women in Culture and Society 35 (4), 2010, 810-820.

Allen, Amy: The Politics of Our Selves. Power, Autonomy, and Gender in Contemporary Critical Theory. New York: Columbia University Press 2008.

Bedorf, Thomas/Röttgers, Kurt (Hg.): Das Politische und die Politik. Berlin: Suhrkamp 2010.

Berlin, Isaiah: „Two Concepts of Liberty“, in: ders.: Liberty. Hg. von Henry Hardy. Oxford: Oxford University Press 2002, 166-217.

Bernstein, Jay: „Rights“. In: Political Concepts. A Critical Lexicon 2012, URL: $<$ https://www.politicalconcepts.org/rights-bernstein/>.

Boyle, James (Hg.): Critical legal studies. Aldershot: Dartmouth 1992.

Bröckling, Ulrich/Feustel, Robert (Hg.): Das Politische denken. Zeitgenössische Positionen. Bielefeld: transcript 2010.

Butler, Judith: „Was ist Kritik. Ein Essay über Foucaults Tugend“, in: Deutsche Zeitschrift für Philosophie 50 (2), 2002, 249-265.

Butler, Judith/ Laclau, Ernesto/Žižek, Slavoj: Contingency, Hegemony, Universality. Contemporary Dialogues on the Left. London: Verso 2000.

Fischer-Lescano, Andreas: Globalverfassung. Die Geltungsbegründung der Menschenrechte. Weilerswist: Velbrück Wissenschaft 2005.

Fitzpatrick, Peter/Hunt, Alan (Hg.): Critical legal studies. Oxford: Blackwell 1990.

Flügel, Oliver/Heil, Reinhard/Hetzel, Andreas (Hg.): Die Rückkehr des Politischen. Demokratietheorien heute. Darmstadt: Wissenschaftliche Buchgesellschaft 2004.

Forst, Rainer: „Ethik und Moral“, in: Wingert, Lutz/Günther, Klaus (Hg.): Die Öffentlichkeit der Vernunft und die Vernunft der Öffentlichkeit. Festschrift für Jürgen Habermas 2001. Frankfurt am Main: Suhrkamp 2001, 344-371.

Foucault, Michel: Wahnsinn und Gesellschaft. Eine Geschichte des Wahns im Zeitalter der Vernunft. Übers. von Ulrich Köppen. Frankfurt am Main: Suhrkamp 1973.

— Die Ordnung der Dinge. Eine Archäologie der Humanwissenschaften. Übers. von Ulrich Köppen. Frankfurt am Main: Suhrkamp 1974.

— Der Wille zum Wissen. Sexualität und Wahrheit 1. Übers. von Ulrich Raulff und Walter Seitter. Frankfurt am Main: Suhrkamp 1983.

— Der Gebrauch der Lüste. Sexualität und Wahrheit 2. Übers. von Ulrich Raulff und Walter Seitter. Frankfurt am Main: Suhrkamp 1989.

— Die Sorge um sich. Sexualität und Wahrheit 3. Übers. von Ulrich Raulff und Walter Seitter. Frankfurt am Main: Suhrkamp 1989. 
— Die Ordnung des Diskurses. Übers. von Walter Seitter. Frankfurt am Main: Fischer 1991.

— Was ist Kritik? Übers. von Walter Seitter. Berlin: Merve 1992.

- Überwachen und Strafen. Die Geburt des Gefängnisses. Übers. von Walter Seitter. Frankfurt am Main: Suhrkamp 1994.

— Geschichte der Gouvernementalität I. Sicherheit, Territorium, Bevölkerung. Übers. von Claudia Brede-Konersmann und Jürgen Schröder. Frankfurt am Main: Suhrkamp 2004.

— „Die Ethik der Sorge um sich als Praxis der Freiheit. Gespräch mit Helmut Becker, Raul Fornet-Betancourt und Alfred Gomez-Müller, 20.01.1984“, in: ders.: Schriften in vier Bänden. Dits et Ecrits. 1980-1988. Bd. 4. Hg. von Daniel Defert und François Ewald und übers. von Michael Bischoff. Frankfurt am Main: Suhrkamp 2005, 875902.

— „Was ist Aufklärung?“, in: ders.: ders.: Schriften in vier Bänden. Dits et Ecrits. 1980_ 1988. Bd. 4. Hg. von Daniel Defert und François Ewald und übers. von Michael Bischoff. Frankfurt am Main: Suhrkamp 2005, 687-707.

— „Was ist Aufklärung? Auszug aus der Vorlesung vom 5. Januar 1983“, in: ders.: Schriften in vier Bänden. Dits et Ecrits. 1980-1988. Bd. 4. Hg. von Daniel Defert und François Ewald und übers. von Michael Bischoff. Frankfurt am Main: Suhrkamp 2005, 837-848.

— Die Geburt der Biopolitik. Geschichte der Gouvernementalität II. Übers. von Jürgen Schröder. Frankfurt am Main: Suhrkamp 2006.

— Die Regierung des Selbst und der anderen. Vorlesung am Collège de France 1982/83. Übers. von Jürgen Schröder. Berlin: Suhrkamp 2009.

Habermas, Jürgen: Faktizität und Geltung. Beiträge zur Diskurstheorie des Rechts und des demokratischen Rechtsstaats. 2. Auflage. Frankfurt am Main: Suhrkamp 1992.

— „Drei normative Modelle der Demokratie“, in: ders.: Die Einbeziehung des Anderen. Studien zur politischen Theorie. Frankfurt am Main: Suhrkamp 1996, 277-292.

— „Zur Legitimation durch Menschenrechte“, in: ders.: Die postnationale Konstellation. Politische Essays. Frankfurt am Main: Suhrkamp 1998, 170-192.

Hardt, Michael/Negri, Antonio: Empire. Die neue Weltordnung. Übers. von Thomas Atzert und Andreas Wirthensohn. Frankfurt am Main: Campus 2002.

Heil, Reinhard/Hetzel, Andreas (Hg.): Die unendliche Aufgabe. Kritik und Perspektiven der Demokratietheorie. Bielefeld: transcript 2006.

Human Rights Committee "Toonen v. Australia, Communication No. 488/1992, 1994, 31.3.1994, URL: <http://www1.umn.edu/humanrts/undocs/html/vws488.htm>.

Inston, Kevin: Rousseau and radical democracy. London: Continuum 2010.

Laclau, Ernesto/Mouffe, Chantal: Hegemonie und radikale Demokratie. Zur Dekonstruktion des Marxismus. 2. Auflage. Übers. von Michael Hintz und Gerd Vorwallner: Wien: Passagen 2000.

—-Mouffe, Chantal: Hegemony and socialist strategy. Towards a radical democratic politics. 2. Auflage. London: Verso 2001. 
Lefort, Claude: „Die Frage der Demokratie“, in: Rödel, Ulrich (Hg.): Autonome Gesellschaft und libertäre Demokratie. Frankfurt am Main: Suhrkamp 1990, 281297.

—/Gauchet, Marcel: „Über die Demokratie. Das Politische und die Instituierung des Gesellschaftlichen“, in: Rödel, Ulrich (Hg.): Autonome Gesellschaft und libertäre Demokratie. Frankfurt am Main: Suhrkamp 1990, 89-122.

Lemke, Thomas: Eine Kritik der politischen Vernunft. Foucaults Analyse der modernen Gouvernementalität. Hamburg: Argument 1997.

Luhmann, Niklas: „Verfassung als evolutionäre Errungenschaft“, in: Rechtshistorisches Journal 9, 1990, 176-220.

Marchart, Oliver: „Politische Theorie als Erste Philosophie. Warum der ontologischen Differenz die politische Differenz zugrunde liegt“, in: Bedorf, Thomas/Röttgers, Kurt (Hg.): Das Politische und die Politik. Berlin: Suhrkamp 2010, 143-158.

— Die politische Differenz. Zum Denken des Politischen bei Nancy, Lefort, Badiou, Laclau und Agamben. Berlin: Suhrkamp 2011.

— Das unmögliche Objekt. Eine postfundamentalistische Theorie der Gesellschaft. Berlin: Suhrkamp 2013.

Menke, Christoph: Kritik der Rechte. Berlin: Suhrkamp 2015.

Mouffe, Chantal: On the political. London: Routledge 2005.

- Über das Politische. Wider die kosmopolitische Illusion. Übers. von Niels Neumaier. Frankfurt am Main: Suhrkamp 2007.

Nussbaum, Martha C.: „Konstruktionen der Liebe, des Begehrens und der Fürsorge“, in: dies.: Konstruktionen der Liebe, des Begehrens und der Fürsorge. Drei philosophische Aufsätze. Übers. von Joachim Schulte. Stuttgart: Reclam 2002, 133163.

Patton, Paul: „Taylor and Foucault on Power and Freedom“, in: Political Studies 37 (2), 1989, 260-276.

Rancière, Jaques: Das Unvernehmen. Politik und Philosophie. Übers. von Richard Steurer. Frankfurt am Main: Suhrkamp 2002.

Rawls, John: Die Idee des politischen Liberalismus. Aufsätze 1978-1989. Hg. von Wilfried Hinsch. Frankfurt am Main: Suhrkamp 1992.

— Politischer Liberalismus. Übers. von Wilfried Hinsch. Frankfurt am Main: Suhrkamp 1998.

Rosenfeld, Michel: Law, Justice, Democracy, and the Clash of Cultures. A Pluralist Account. Cambridge/New York: Cambridge University Press 2011.

Saar, Martin: Genealogie als Kritik. Geschichte und Theorie des Subjekts nach Nietzsche und Foucault. Frankfurt: Campus 2007.

Schubert, Karsten: „Langer Weg zur sexuellen Selbstbestimmung. Der Schutz von LSBTI durch die Vereinten Nationen“, in: Vereinte Nationen 61 (5), 2013, 216-222.

— „Der letzte Universalismus. Kontingenz, Konflikt und normative Demokratietheorie“. Schwerpunktbeitrag 2017, URL: <https://philosophie- 
indebate.de/2995/schwerpunktbeitrag-der-letzte-universalismus-kontingenzkonflikt-und-normative-demokratietheorie/>.

— Freiheit als Kritik. Sozialphilosophie nach Foucault. Bielefeld: transcript 2018.

— „Die christlichen Wurzeln der Kritik. Wie Foucaults Analysen der Kirchenväter neues Licht auf die Debatte um Macht und Freiheit werfen. Rezension von Michel Foucault: Die Geständnisse des Fleisches. Sexualität und Wahrheit 4. Frankfurt a. M.: Suhrkamp 2019“, in: Zeitschrift für philosophische Literatur 7 (2), 2019, 60-71.

— „Der letzte Universalismus. Kontingenz, Konflikt und normative Demokratietheorie“, in: Oliver Flügel-Martinsen/Franziska Martinsen/Martin Saar (Hg.): Das Politische (in) der politischen Theorie. Baden-Baden: Nomos 2020. [im Erscheinen]

— „Sklavenmoral und 'Political Correctness'. Linksnietzscheanische Kritik eines rechtsnietzscheanischen Kampfbegriffs“, in: Leviathan 48 (1), 2020. [im Erscheinen]

Stein, Edward: „Born That Way? Not A Choice?: Problems with Biological and Psychological Arguments for Gay Rights“, in: Cardozo Legal Studies Research Paper No. 223, 2008.

- „Sexual Orientations, Rights, and the Body: Immutability, Essentialism, and Nativism“, in: Social Research 78 (2), 2011, 633-658.

Teubner, Gunther: „Ökonomie der Gabe - Positivität der Gerechtigkeit: Gegenseitige Heimsuchungenvon System und différance“, in: Albrecht Koschorke/Cornelia Vismann (Hg.): Widerstände der Systemtheorie. Kulturtheoretische Analysen zum Werk von Niklas Luhmann 1999, Berlin: Akademie Verlag 1999, 199-212.

- (Hg.): Nach Jacques Derrida und Niklas Luhmann: zur (Un-)Möglichkeit einer Gesellschaftstheorie der Gerechtigkeit. Stuttgart: Lucius \& Lucius 2008.

Williams, Patricia J.: The Alchemy of Race and Rights. Diary of a Law Professor. Cambridge/Massachusetts: Harvard University Press 1991. 